\title{
THE MINIMAL FAITHFUL DEGREE OF A SEMILATTICE OF GROUPS
}

\author{
DAVID EASDOWN
}

(Received 5 September, 1986)

Communicated by T. E. Hall

\begin{abstract}
This paper constructs a minimal faithful representation of a semilattice of groups by partial transformations. The solution is expressed in terms of join irreducible elements of the semilattice and minimal faithful representations of groups with respect to certain normal subgroups.
\end{abstract}

1980 Mathematics subject classification (Amer. Math. Soc.): 20 M 20, 20 M 30.

\section{Introduction}

It is natural to ask, for a given semigroup $S$, what is the least size of a set $X$ for which $S$ may be faithfully represented by partial transformations of $X$. This question, among others, was posed by Schein in [10, Problem 45]. In this paper, the problem is solved for any given finite semilattice of groups. The solution is expressed in terms of joint irreducible elements of the semilattice and a slight generalization of a well-known result for groups. In the final section an example is given which illustrates the construction embodied in the proof of the main theorem.

(C) 1988 Australian Mathematical Society $0263-6115 / 88 \$ A 2.00+0.00$ 


\section{Preliminaries}

Standard terminology and basic results relating to inverse semigroups, and representations by the symmetric inverse semigroup in particular, as given by Howie [4], Petrich [8] or Clifford and Preston [9] will be assumed.

The symmetric group, symmetric inverse semigroup and semigroup of partial mappings on a set $X$ will be denoted by $\mathscr{G}_{X}, \mathscr{I}_{X}$ and $\mathscr{P F}_{X}$ respectively. The identity relation on $X,\{(x, x) \mid x \in X\}$, will be denoted by id $X$.

If $\psi: S \rightarrow T$ is a homomorphism between semigroups $S$ and $T$, denote the congruence

$$
\{(x, y) \in S \times S \mid x \psi=y \psi\}
$$

by $\psi \circ \psi^{-1}$. If further $S$ is a group with identity $e$, denote the congruence class containing $e$ by $\operatorname{ker} \psi$. Both $\psi \circ \psi^{-1}$ and ker $\psi$ are known in the literature as the kernel of $\psi$, and are related by

$$
\psi \circ \psi^{-1}=\left\{(x, y) \in S \times S \mid x y^{-1} \in \operatorname{ker} \psi\right\} .
$$

If $\psi: S \rightarrow \mathscr{P} \mathscr{T}_{X}$ and $\chi: S \rightarrow \mathscr{P} \mathscr{F}_{Y}$ are representations of a semigroup $S$, where $X$ and $Y$ are disjoint sets, define the direct sum $\psi \oplus \chi$ of $\psi$ and $\chi$ to be the representation

$$
\psi \oplus \chi: S \rightarrow \mathscr{P} \mathscr{T}_{X \cup Y}
$$

where, for $x \in S$,

$$
x(\psi \oplus \chi)=(x \psi) \cup(x \chi)
$$

Clearly,

$$
(\psi \oplus \chi) \circ(\psi \oplus \chi)^{-1}=\left(\psi \circ \psi^{-1}\right) \cap\left(\chi \circ \chi^{-1}\right),
$$

so, if $S$ is a group,

$$
\operatorname{ker}(\psi \oplus \chi)=\operatorname{ker} \psi \cap \operatorname{ker} \chi
$$

If $\psi: S \rightarrow \mathscr{P} \mathscr{T}_{X}$ is a representation, call $|X|$ the degree of $\psi$, and write $|X|=$ degree $(\psi)$. Call $\psi$ effective if each element of $X$ is in the domain or range of $s \psi$ for some $s \in S$.

If $S$ is a finite semigroup, define the minimal faithful degree $\mu(S)$ of $S$ to be the least non-negative integer $n$ such that $S$ can be embedded in $\mathscr{P} \mathscr{G}_{X}$ where $|X|=n$. Further, if $\phi: S \rightarrow \mathscr{P} \mathscr{F}_{X}$ is an embedding, say $\phi$ realizes $\mu(S)$ and call $\phi$ a minimal faithful representation. Note, because of the extended right regular representation, $\mu(S)$ exists and is bounded by $|S|+1$. Note also $\mu(S)=0$ if and only if $|S|=1$.

The following theorem shows that for a given finite inverse semigroup $S, \mu(S)$ may always be realized by a representation into $\mathscr{I}_{X}$, where $|X|=\mu(S)$, so in that case there is no advantage in using partial transformations which are not one-one. 
THEOREM 1 ([8, IV.5.9], [7, II.8.4], [11], [9]). Let $S$ be an inverse semigroup and $\psi: S \rightarrow \mathscr{P S}_{X}$ a faithful representation. For $s \in S$, define

$$
s \bar{\psi}=\left.s \psi\right|_{\text {range }\left(s^{-1} \phi\right)} .
$$

Then $\bar{\psi}: S \rightarrow \mathscr{I}_{X}$ is a faithful representation.

PROPOSITION 2. Any effective representation of a group by partial one-one mappings is by permutations.

Proof. Let $G$ be a group with identity $e$, and $\psi: G \rightarrow \mathscr{I}_{X}$ an effective representation. Then $e \psi=\mathrm{id}_{X}$, so for each $g \in G, X$ is both the domain and range of $g \psi$. Thus each $g \psi$ is a permutation.

COROLlaRY 3. Any minimal faithful representation of a finite group is by permutations.

ProOF. Let $\psi$ be a minimal faithful representation of a finite group $G$. By Theorem $1, \bar{\psi}$ is also faithful and $g \bar{\psi}$ is one-one for each $g \in G$. But $\bar{\psi}$ must be effective, for otherwise $\psi$ would not be minimal. Hence, by Proposition 2, each $g \bar{\psi}$ is a permutation, so $\psi=\bar{\psi}$. This complete the proof.

If $H$ is a subgroup of a group $G$, define the core of $H$, core $(H)$, to be the largest normal subgroup of $G$ contained in $H$. Thus core $(H)$ is the kernel of the representation of $G$ induced by right multiplication of cosets of $H$ in $G$. Denote the index of $H$ in $G$ by $|G: H|$. The following solution is well known (see, for example, [5]).

THEOREM 4. Let $G$ be a finite non-trivial group with identity e. Then $\mu(G)$ is the least positive integer $n$ such that, for some subgroups $H_{1}, \ldots, H_{m}$ of $G$,

$$
\bigcap_{i=1}^{m} \operatorname{core}\left(H_{i}\right)=\{e\}
$$

and

$$
\sum_{i=1}^{m}\left|G: H_{i}\right|=n .
$$

The following definition will be useful later. If $G$ is a finite group with normal subgroup $N$ and identity $e$, let $\mu(G \mid N)$, the minimal degree of $G$ with respect to $N$, be the least non-negative integer $n$ such that there exists a representation $\psi: G \rightarrow \mathscr{G}_{X}$ satisfying $|X|=n$ and $\operatorname{ker} \psi \cap N=\{e\}$. We say $\psi$ realizes $\mu(G \mid N)$. Thus, for example, $\mu(G)=\mu(G \mid G)$ and $\mu(G \mid\{e\})=0$.

The following is an immediate generalization of Theorem 4 . 
THEOREM 5. Let $G$ be a finite group with identity e and non-trivial normal subgroup $N$. Then $\mu(G \mid N)$ is the least positive integer $n$ such that, for some subgroups $H_{1}, \ldots, H_{m}$ of $G$,

$$
N \cap \bigcap_{i=1}^{m} \operatorname{core}\left(H_{i}\right)=\{e\}
$$

and

$$
\sum_{i=1}^{m}\left|G: H_{i}\right|=n .
$$

In describing semilattices of groups, the notation and basic results (originally due to Clifford [1]) as described in any of [4, IV], [8, II.2] or $[2,4.2]$ will be assumed, except that, if $S$ is the semilattice of groups $\bigcup\left\{G_{e} \mid e \in E\right\}$, the identity of each group $G_{e}$ will be identified with $e$. Thus if $e, f \in E$ and $f \leq e$, then

$$
\phi_{e, f}: G_{e} \rightarrow G_{f}
$$

is the homomorphism defined by, for $g \in G_{e}$,

$$
g \phi_{e, f}=g f,
$$

where $g f$ is the product in $S$.

If $E$ is a semilattice, the symbol $\vee$ denotes the supremum, when it exists, with respect to the partial order $\leq$ of $E$. If $e, f \in E$, then $e<f$ means $e \leq f$ and $e \neq f$. If $E$ has a least element $e$, which exists when $E$ is finite, then $e$ is called the zero of $E$. Call an element $e$ of $E$ join irreducible if $e$ is non-zero and, for $f, g \in E$,

$$
e=f \vee g \quad \text { implies } \quad e=f \text { or } e=g .
$$

It is easy to see that, for a finite semilattice $E$, an element $e$ is joint irreducible if and only if $e$ has a unique immediate predecessor, that is, element $f$ for which $f<e$, and $g<e$ implies $g \leq f$.

The following is a trivial but useful observation.

LEMMA 6. Let $P$ be a finite partially ordered set with $n$ elements. Then $P=\left\{p_{1}, \ldots, p_{n}\right\}$ may be listed so that

$$
p_{i} \leq p_{j} \quad \text { implies } i \leq j .
$$

\section{Semilattices of groups}

The main aim of this paper is to prove the following: 
THEOREM 7. Let $S$ be a finite semilattice of groups, so there is a semilattice $E$ and collection of groups $\left\{G_{e} \mid e \in E\right\}$ for which $S=\bigcup\left\{G_{e} \mid e \in E\right\}$. For each $e \in E$, put

$$
N_{e}= \begin{cases}G_{e} & \text { if } e \text { is the zero of } E, \\ \bigcap_{f<e} \operatorname{ker} \phi_{e, f} & \text { if } e \text { is nonzero. }\end{cases}
$$

Let $J(S)=\left\{e \in E \mid e\right.$ is join irreducible and $\operatorname{ker} \phi_{e, f}=\{e\}$ where $f$ is the unique immediate predecessor of $e\}$. Then

$$
\mu(S)=\sum_{e \in E} \mu\left(G_{e} \mid N_{e}\right)+|J(S)|
$$

For example, if $S$ is a group then the statement of the theorem reduces to the triviality $\mu(S)=\mu(S \mid S)$. If $S$ is a semilattice, then $\mu(S)$ is the number of join irreducible elements of $S$, a result obtained in [3].

The proof of Theorem 7 will be by induction. First a sequence of lemmas is proven, in which it is assumed $S=\bigcup\left\{G_{f} \mid f \in E\right\}$ is a finite semilattice of groups, and $e$ is a non-zero maximal element of $E$, that is, $e \leq f$ implies $e=f$. Put $T=S \backslash G_{e}$. By the maximality of $e, T$ is an ideal of $S$, so the following two lemmas follow immediately.

Lemma 8. Define $\xi: S \rightarrow \mathscr{I}_{\{e\}}$ by, for $x \in S$,

$$
x \xi= \begin{cases}\operatorname{id}_{\{e\}} & \text { if } x \in G_{e}, \\ \varnothing & \text { if } x \in T\end{cases}
$$

Then $\xi$ is a representation of $S$ and

$$
\xi \circ \xi^{-1}=\left(G_{e} \times G_{e}\right) \cup(T \times T) .
$$

LEMMA 9. Let $\zeta: G_{e} \rightarrow \mathscr{G}_{X}$ be a representation of $G_{e}$ which realizes $\mu\left(G_{e} \mid N_{e}\right)$. Define $\varsigma_{1}: S \rightarrow \mathscr{I}_{X}$ by, for $x \in S$,

$$
x \varsigma_{1}= \begin{cases}x_{\zeta} & \text { if } x \in G_{e}, \\ \varnothing & \text { if } x \in T\end{cases}
$$

Then $\zeta$ is a representation of $S$ and

$$
\zeta_{1} \circ \varsigma_{1}^{-1}=\left\{(x, y) \in G_{e} \times G_{e} \mid x y^{-1} \in \operatorname{ker} \zeta\right\} \cup(T \times T) .
$$

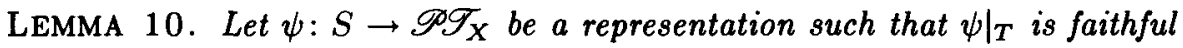
and effective. Put $\psi_{1}=\left.\psi\right|_{G_{e}}$. Then $\operatorname{ker} \psi_{1}=N_{e}$ (where $N_{e}$ is defined in Theorem 7).

Proof. Suppose $g \in \operatorname{ker} \psi_{1}$, so $g \psi=e \psi$. Hence, if $f<e, g\left(\phi_{e, f} \psi\right)=$ $(g f) \psi=g \psi f \psi=e \psi f \psi=(e f) \psi=e\left(\phi_{e, f} \psi\right)$, so $g \in \operatorname{ker}\left(\phi_{e, f} \psi\right)=\operatorname{ker} \phi_{e, f}$, since $\left.\psi\right|_{T}$ is faithful. This shows $\operatorname{ker} \psi_{1} \subseteq N_{e}$. 
Conversely, suppose $g \in N_{e}$, so $g f=e f=f$ for all $f<e$. Let $\alpha \in$ domain $(e \psi)$, so, since $\left.\psi\right|_{T}$ is effective, there is some $e^{\prime} \in E, e^{\prime} \neq e$, for which $\alpha \in \operatorname{domain}\left(e^{\prime} \psi\right)$. Hence $\alpha \in \operatorname{domain}\left(e e^{\prime} \psi\right)$ and $e e^{\prime}<e$. Thus

$$
\alpha(g \psi)=\alpha\left(e e^{\prime} \psi\right)(g \psi)=\alpha\left(e e^{\prime} g \psi\right)=\alpha\left(e e^{\prime} \psi\right)=\alpha .
$$

This shows $g \psi=e \psi$, so $g \in \operatorname{ker} \psi_{1}$. Hence $N_{e} \subseteq \operatorname{ker} \psi_{1}$, and the proof is complete.

LEMmA 11. Let $\psi: T \rightarrow \mathscr{I}_{X}$ be a faithful and effective representation. Define $\chi: S \rightarrow \mathscr{I}_{X}$ by

$$
x \chi= \begin{cases}x \psi & \text { if } x \in T, \\ \bigcup_{f<e}(x f) \psi & \text { if } x \in G_{e} .\end{cases}
$$

Then $\chi$ is a well-defined representation. If $e$ is not join irreducible then

$$
\chi \circ \chi^{-1}=\left\{(x, y) \in G_{e} \times G_{e} \mid x y^{-1} \in N_{e}\right\} \cup \operatorname{id}_{T} .
$$

If $e$ is join irreducible with unique immediate predecessor $e^{\prime}$ then

$$
\chi \circ \chi^{-1}=\left\{(x, y) \in G_{e} \times G_{e} \mid x y^{-1} \in \operatorname{ker} \phi_{e, e^{\prime}}\right\} \cup \operatorname{id}_{T} \cup\left\{\left(x, x e^{\prime}\right) \mid x \in G_{e}\right\} .
$$

ProOF. We first show $\chi$ is well defined. If $x \in T$, then $x \chi=x \psi \in \mathscr{I}_{X}$. Suppose $x \in G_{e}$. We show $x \chi$ is a partial mapping. Suppose $(\alpha, \beta)$ and $(\alpha, \gamma) \in \bigcup_{f<e}(x f) \psi$, so for some $f_{1}, f_{2}<e, \alpha\left(x f_{1} \psi\right)=\beta \quad$ and $\quad \alpha\left(x f_{2} \psi\right)=\gamma$. Hence $\alpha \in \operatorname{domain}\left(f_{1} \psi\right) \cap \operatorname{domain}\left(f_{2} \psi\right)=\operatorname{domain}\left(f_{1} f_{2} \psi\right)$ Thus, $\beta=\alpha\left(x f_{1} \psi\right)=$ $\alpha\left(f_{1} f_{2} \psi\right)\left(x f_{1} \psi\right)=\alpha\left(f_{1} f_{2} x \psi\right)=\alpha\left(f_{1} f_{2} \psi\right)\left(x f_{2} \psi\right)=\alpha\left(x f_{2} \psi\right)=\gamma$, so $x \chi$ is a partial mapping.

We shown $x \chi$ is one-one. Suppose $(\alpha, \gamma)$ and $(\beta, \gamma) \in x \chi$, so for some $f_{1}, f_{2}<$ $e, \alpha\left(x f_{1} \psi\right)=\gamma$ and $\beta\left(x f_{2} \psi\right)=\gamma$, so $\gamma\left(x^{-1} f_{1} \psi\right)=\alpha$ and $\gamma\left(x^{-1} f_{2} \psi\right)=\beta$. Applying the previous argument, we get $\alpha=\beta$. This shows $x \chi \in \mathscr{I}_{X}$.

We show $\chi$ is a homomorphism. Suppose $x, y \in G_{e}$, so $x y \in G_{e}$. Let $\alpha \in$ $\operatorname{domain}(x y \chi)$, so $\alpha \in \operatorname{domain}(x y f \psi)$ for some $f<e$. Hence

$$
\alpha(x y \chi)=\alpha(x y f \psi)=\alpha(x f \psi)(y f \psi)=\alpha(x \chi)(y \chi) .
$$

Conversely, suppose $\alpha \in \operatorname{domain}(x \chi y \chi)$, so $\alpha \in \operatorname{domain}(x f \psi)$ for some $f<e$ and $\alpha(x f) \psi \in \operatorname{domain}(y g \psi)$ for some $g<e$. But $f g<e$, and $\alpha \in \operatorname{domain}(x f \psi y g \psi)=$ domain $(x y f g \psi)$, which shows $\alpha \in \operatorname{domain}(x y \chi)$. This shows $(x y) \chi=x \chi y \chi$. The case when one of $x, y$ lies in $G_{e}$ and the other in $T$ is similar, and the case both $x, y$ lie in $T$ is covered since $\left.\chi\right|_{T}=\psi$. Thus $\chi$ is a homomorphism.

Now we calculate $\chi \circ \chi^{-1}$. Suppose $x \chi=y \chi$. If $x, y \in T$ then $x=y$, since $\left.\chi\right|_{T}=\psi$ and $\psi$ is faithful.

If $x, y \in G_{e}$ then $x y^{-1} \in N_{e}$, by Lemma 10: If $e$ has a unique immediate predecessor $e^{\prime}$, then $N_{e}=\operatorname{ker} \phi_{e, e^{\prime}}$, since if $f<e$ then $f \leq e^{\prime}$, so

$$
\operatorname{ker} \phi_{e, f}=\operatorname{ker}\left(\phi_{e, e^{\prime}} \phi_{e^{\prime}, f}\right) \supseteq \operatorname{ker} \phi_{e, e^{\prime}} \text {. }
$$


Suppose now $x \in G_{e}$ and $y \in T$, so $\bigcup_{f<e}(x f) \psi=y \psi$. For some $g \in E, y$ lies in $G_{g}$, so

$$
\operatorname{domain}(y \psi)=\operatorname{domain}(g \psi)=\bigcup_{f<e} \operatorname{domain}(f \psi) .
$$

Hence, if $f<e$ then $f \leq g$, so $f \leq e g$. But $e \neq e g$, since $e$ is maximal in $E$, so $e g$ is the unique immediate predecessor of $e$, which shows $e$ is join irreducible. Thus $\bigcup_{f<e}(x f) \psi=(x e g) \psi$, so $y=x e g$, since $\psi$ is faithful. This completes the proof of the lemma.

We now return to the more general hypotheses of Theorem 7 .

ProOF OF ThEOREM 7 . By Lemma 6 we may suppose $E=\left\{e_{1}, \ldots, e_{n}\right\}$ where $e_{i} \leq e_{j}$ implies $i \leq j$. For $e_{k} \in E$, put $S_{k}=\bigcup_{i \leq k} G_{e_{i}}$, so $S=S_{n}$ and each $S_{k}$ is a subsemigroup of $S$ with semilattice $\left\{e_{1}, \ldots, e_{k}\right\}$, of which $e_{k}$ is a maximal element.

Put $M_{k}=\sum_{i=1}^{k} \mu\left(G_{e_{i}} \mid N_{e_{i}}\right)+\left|J\left(S_{k}\right)\right|$. We show by induction that $\mu\left(S_{k}\right)=M_{k}$ for $k=1$ to $n$.

Observe that $S_{1}=G_{e_{1}}$ and $N_{e_{1}}=G_{e_{1}}$, so $\mu\left(S_{1}\right)=\mu\left(G_{e_{1}}\right)=\mu\left(G_{e_{1}} \mid N_{e_{1}}\right)=$ $M_{1}$, which starts the induction.

Suppose now $k<n$ and $\mu\left(S_{k}\right)=M_{k}$, so there is some faithful representation $\psi: S_{k} \rightarrow \mathscr{I}_{X}$ where $|X|=M_{k}$. Define $\chi: S_{k+1} \rightarrow \mathscr{I}_{X}$ by

$$
x \chi= \begin{cases}x \psi & \text { if } x \in S_{k}, \\ \bigcup_{f<e_{k+1}}(x f) \psi & \text { if } x \in G_{e_{k+1}} .\end{cases}
$$

By Lemma 11, $\chi$ is a representation. We extend $\chi$ to a faithful representation of $S_{k+1}$ of the appropriate degree, but the argument splits into two cases.

Case (i). Suppose $e_{k+1} \notin J(S)$. Let $\varsigma: G_{e_{k+1}} \rightarrow \mathscr{G}_{Y}$ be a representation of $G_{e_{k+1}}$ which realizes $\mu\left(G_{e_{k+1}} \mid N_{e_{k+1}}\right)$, and suppose $X$ and $Y$ are disjoint. Define $\varsigma_{1}$ as in Lemma 9, and put $\psi_{1}=\chi \oplus \varsigma_{1}$, so

$$
\psi_{1} \circ \psi_{1}^{-1}=\left(\chi \circ \chi^{-1}\right) \cap\left(\zeta_{1} \circ \zeta_{1}^{-1}\right)=\mathrm{id}_{S_{k+1}},
$$

by Lemmas 9 and 11, since $\operatorname{ker} \zeta \cap N_{e_{k+1}}=\left\{e_{k+1}\right\}$. Thus $\psi_{1}$ is faithful. Also, $|X \cup Y|=M_{k}+\mu\left(G_{e_{k+1}} \mid N_{e_{k+1}}\right)=M_{k+1}$.

Case (ii). Suppose $e_{k+1} \in J(S)$, and let $e^{\prime}$ be the unique immediate predecessor of $e_{k+1}$. Let $\xi: S_{K+1} \rightarrow \mathscr{I}_{\left\{e_{k+1}\right\}}$ be the representation defined in Lemma 8, where it may be assumed $e_{k+1} \notin X$. Put $\psi_{1}=\chi \oplus \xi$. Note that $N_{e_{k+1}}=\operatorname{ker} \phi_{e_{k+1}, e^{\prime}}=\left\{e_{k+1}\right\}$, so by Lemma 11 ,

$$
\chi \circ \chi^{-1}=\operatorname{id}_{G_{e_{k+1}}} \cup \operatorname{id}_{T} \cup\left\{\left(x, x e^{\prime}\right) \mid x \in G_{e_{k+1}}\right\} .
$$

Hence, by Lemma $8, \psi_{1} \circ \psi_{1}^{-1}=i \mathrm{~d}_{S_{k+1}}$, so $\psi_{1}$, is Iaithiul. A Aso, $\left.\nmid \mathrm{X} \cup\left\{e_{k+1}\right\}\right\rangle=$ $M_{k}+1=M_{k+1}$. 
In both cases a faithful representation of $S$ of degree $M_{k+1}$ has been exhibited, so $\mu\left(S_{k+1}\right) \leq M_{k+1}$.

Suppose now $\theta: S_{k+1} \rightarrow \mathscr{I}_{Z}$ is a faithful representation. We show $|Z| \geq$ $M_{k+1}$. Let $\theta_{1}$ be the effective part of $\left.\theta\right|_{S_{k}}$, so the degree of $\theta_{1}$ is at least $M_{k}$, by the inductive hypothesis.

Case (i). Suppose $e_{k+1} \notin J(S)$.

Let $W$ be the set of elements of $Z$ which are deleted when $\theta_{1}$ is formed, and let $\theta_{W}$ ad $\theta_{Z / W}$ be the restrictions of $\left.\theta\right|_{G_{e_{k+1}}}$ to $W$ and $Z \backslash W$ respectively, so

$$
\left.\theta\right|_{G_{e_{k+1}}}=\theta_{W} \oplus \theta_{Z \backslash W} .
$$

Since $\theta$ is faithful, $\operatorname{ker} \theta_{W} \cap \operatorname{ker} \theta_{Z \backslash W}=\left\{e_{k+1}\right\}$. By Lemma $10, \operatorname{ker} \theta_{Z \backslash W}=N_{e_{k+1}}$. Hence the degree of $\theta_{W}$, which is $|W|$, must be at least $\mu\left(G_{e_{k+1}} \mid N_{e_{k+1}}\right)$. This shows the degree of $\theta$ is at least $M_{k}+\mu\left(G_{e_{k+1}} \mid N_{e_{k+1}}\right)=M_{k+1}$.

Case (ii). Suppose $e_{k+1} \in J(S)$, so $M_{k+1}=M_{k}+1$. Hence it suffices to show $\operatorname{degree}(\theta)>\operatorname{degree}\left(\theta_{1}\right)$.

Suppose $\operatorname{degree}(\theta)=\operatorname{degree}\left(\theta_{1}\right)$. Let $\alpha \in \operatorname{domain}\left(e_{k+1} \theta\right)$, so also $\alpha \in$ $\operatorname{domain}(f \theta)$ for some idempotent $f$ in $S_{k}$. But $e_{k+1} f<e_{k+1}$, so $e_{k+1} f \leq e^{\prime}$, where $e^{\prime}$ is the unique immediate predecessor of $e$. Hence $\alpha \in \operatorname{domain}\left(e^{\prime} \theta\right)$. Thus domain $\left(e_{k+1} \theta\right) \subseteq$ domain $\left(e^{\prime} \theta\right)$, so $e_{k+1} \leq e^{\prime}$, which is impossible. Hence $\operatorname{degree}(\theta) \geq M_{k+1}$.

Thus we have shown $\mu\left(S_{k+1}\right)=M_{k+1}$, so Theorem 7 follows by induction.

\section{Example}

The proof of Theorem 7 is algorithmic in the sense that given the appropriate representations for the groups involved, one is shown how to paste these together to obtain a minimal faithful representation for the union.

The following example uses only abelian groups, but illustrates the salient features of the construction. Calculations of minimal faithful degrees of abelian groups are undertaken in [6] and [5].

Let $E$ be the following semilattice.

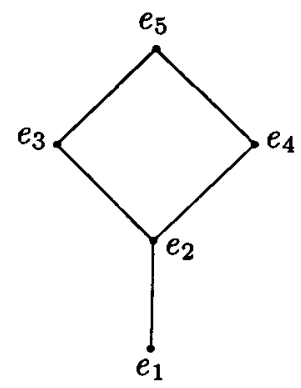


Denote the cyclic group with $n$ elements by $C_{n}$. Let $S=\bigcup\left\{G_{e_{i}} \mid i=1\right.$ to 5$\}$ where

$$
\begin{aligned}
& G_{e_{1}}=\left\langle x \mid x^{4}=e_{1}\right\rangle \cong C_{4} \\
& G_{e_{2}}=\left\langle y \mid y^{2}=e_{2}\right\rangle \cong C_{2} ; \\
& G_{e_{3}}=\left\langle w \mid w^{4}=e_{3}\right\rangle \cong C_{4} ; \\
& G_{e_{4}}=\left\langle v \mid v^{8}=e_{4}\right\rangle \cong C_{8} ; \\
& G_{e_{5}}=\left\langle t, u \mid t^{2}=u^{8}=e_{5}, t u=u t\right\rangle \cong C_{2} \times C_{8} .
\end{aligned}
$$

The multiplication of $S$ is determined by homomorphisms $\phi_{e, f}$ where $f \leq e$. Consider the following, which are induced by the given actions on generators, and from which all the other homomorphisms can be deduced:

$$
\begin{aligned}
& \phi_{e_{2}, e_{1}}: y \rightarrow x^{2} ; \\
& \phi_{e_{3}, e_{2}}: w \rightarrow y ; \\
& \phi_{e_{4}, e_{2}}: v \rightarrow y ; \\
& \phi_{e_{3}, e_{3}}: t \rightarrow w^{2}, u \rightarrow w ; \\
& \phi_{e_{3}, e_{4}}: t \rightarrow e_{4}, u \rightarrow v .
\end{aligned}
$$

It is easy to calculate that $N_{e_{1}}=G_{e_{1}}, N_{e_{2}}=\left\{e_{2}\right\}, N_{e_{3}}=\left\langle w^{2}\right\rangle, N_{e_{4}}=\left\langle v^{4}\right\rangle$, $N_{e_{5}}=\left\langle t u^{2}\right\rangle \cap\langle t\rangle=\left\{e_{5}\right\}, \mu\left(G_{e_{1}} \mid N_{e_{1}}\right)=4, \mu\left(G_{e_{2}} \mid N_{e_{2}}\right)=0, \mu\left(G_{e_{3}} \mid N_{e_{3}}\right)=4$, $\mu\left(G_{e_{4}} \mid N_{e_{4}}\right)=8$ and $\mu\left(G_{e_{5}} \mid N_{e_{5}}\right)=0$. Also $J(S)=\left\{e_{2}\right\}$. By Theorem 7, $\mu(S)=4+0+4+8+0+1=17$. Explicitly, $S$ is isomorphic to the union of the following subgroups of $\mathscr{I}_{\{1, \ldots, 17\}}$, where the usual cyclic notation is employed for a permutation, except that all singleton cycles are included to indicate the domain. The groups are listed in the order of construction, following the method of the previous section:

$$
\begin{aligned}
& G_{e_{1}} \cong\langle(1234)\rangle ; \\
& G_{e_{2}} \cong\langle(13)(24)(5)\rangle ; \\
& G_{e_{3}} \cong\langle(13)(24)(5)(6789)\rangle ; \\
& G_{e_{4}} \cong\langle(13)(24)(5)(1011121314151617)\rangle ; \\
& G_{e_{3}} \cong\langle(1) \ldots(5)(68)(79)(10) \ldots(17),(13)(24)(5)(6789)(10 \ldots 17)\rangle .
\end{aligned}
$$

\section{References}

[1] A. H. Clifford, 'Semigroups admitting relative inverses', Ann. of Math. 42 (1941), 10371049.

[2] A. H. Clifford and G. B. Preston, The algebraic theory of semigroups (Math. Surveys No. 7, Amer. Math. Soc., Providence, R.I., Vol I (1961), Vol. II (1967)).

[3] D. Easdown, 'The minimal faithful degree of a fundamental inverse semigroup', Bull. Austral. Math. Soc. 35 (1987), 373-378. 
[4] J. M. Howie, An introduction to semigroup theory (London Mathematical Society Monographs 7, Academic Press, London, 1976).

[5] D. L. Johnson, 'Minimal permutation representations of finite groups', Amer. J. Math. 93 (1971), 857-866.

[6] G. I. Karpilovsky, 'The least degree of a faithful representation of abelian groups', Vestnik Har'kov. Gos. Univ. 53 (1970), 107-115.

[7] E. S. Ljapin, Semigroups (Translations of Mathematical Monographs, vol. 3, Amer. Math. Soc., Providence, R.I., 1974).

[8] Mario Petrich, Inverse semigroups (Pure and Applied Mathematics, John Wiley \& Sons, 1984).

[9] G. B. Preston, 'Representations of inverse semigroups by one-to-one partial transformations of a set', Semigroup Forum 6 (1973), 240-245; Addendum: ibid 8 (1974), 277.

[10] L. N. Shevrin, 'The Sverdlovsk tetrad', Semigroup Forum 4 (1972), 274-280.

[11] V. V. Vagner, 'The theory of generalized heaps and generalized groups', Mat. $S b .32$ (1953), 545-632.

Department of Mathematics

University of Western Australia

Nedlands, 6009

Australia 